(2)

\title{
Reduced dose maintenance eculizumab in atypical hemolytic uremic syndrome (aHUS): an update on a previous case report
}

This article was published in the following Dove Press journal:

Clinical Pharmacology: Advances and Applications

31 October 20 I I

Number of times this article has been viewed

\section{Maro Ohanian \\ Christian Cable \\ Kathleen Halka}

Department of Hematology/Oncology, Scott \& White Healthcare and The

Texas A\&M Health Science Center College of Medicine, Temple, TX, USA

Correspondence: Maro Ohanian Department of Hematology/Oncology, Scott \& White Healthcare, 240I South 3 Ist Street Temple, TX 76508, USA

$\mathrm{Tel}+\mathrm{I} 28 \mid 2171974$

Fax + I $25477 \mid 4890$

Email ohanianus@yahoo.com
Objective: To describe how maintenance eculizumab sustains improved renal function in severe atypical hemolytic uremic syndrome (aHUS).

Case report: A previously described 50-year-old woman with aHUS had a remarkable recovery with eculizumab, which safely reversed profound neurologic damage and eliminated the need for dialysis. Her recovery has been sustained on long-term eculizumab treatment. She initially received eculizumab $900 \mathrm{mg}$ weekly for four doses. On week 5 she commenced maintenance therapy starting at $1200 \mathrm{mg}$ every 2 weeks. Due to nausea and vomiting at that dose, the maintenance dosing was reduced to $600 \mathrm{mg}$ weekly, beginning on dose seven. After receiving $600 \mathrm{mg}$ weekly for nine doses, eculizumab was then reduced to $600 \mathrm{mg}$ every 2 weeks, with continued improvement in renal function. This dosing is lower than the usual 1200 mg every 2 weeks described in the adult literature and used in current clinical trials of aHUS.

Conclusion: Six months after the initial diagnosis, our patient continues to have improved renal function on maintenance doses of eculizumab as low as $600 \mathrm{mg}$ every 2 weeks.

Keywords: eculizumab, aHUS, thrombotic microangiopathy, atypical hemolytic uremic syndrome

\section{Introduction}

Atypical Hemolytic Uremic Syndrome (aHUS) involves congenital or acquired chronic systemic alternative complement pathway dysregulation. This causes reduced host cell protection, dysfunctional complement activation of platelets and endothelial cells, inflammation, and thrombotic microangiopathy. ${ }^{1,2}$ Terminal complement inhibition with eculizumab safely reversed our patient's neurologic changes and eliminated the need for dialysis.

While the optimal duration and frequency of therapy remains to be determined (and varies in the literature), complete and sustained terminal complement inhibition has been considered necessary for adequate control of aHUS. ${ }^{3}$ Previous publications have indicated that long-term eculizumab therapy helps avoid aHUS relapse and maintenance therapy is used in current aHUS clinical trials. ${ }^{1-6}$

Eculizumab has been reported to be safe and effective in aHUS, a condition of chronic systemic uncontrolled complement activation. ${ }^{4-15}$ Eculizumab is a highaffinity humanized monoclonal anti-C5 antibody that blocks terminal complement activity. Eculizumab binds to and blocks cleavage of the terminal complement protein C5 into its pro-inflammatory, prothrombotic, and lytic products: C5a and the cytotoxic 
membrane-attack complex C5b-9. Upstream components of complement, most notably $\mathrm{C} 3 \mathrm{~b}$, are left intact. $3,7,15,16$

While eculizumab is not yet FDA approved for aHUS, this update describes how long term eculizumab maintains and improves renal function, despite being used at doses lower than currently used in clinical trials. This update also reports the results of our patient's genetic and antibody tests, all of which were normal.

\section{Case summary and update}

A previously described 50-year-old Caucasian woman was transferred to our intensive care unit with sepsis, pancolitis, acute renal failure, and thrombocytopenia. Low complement levels were noted: C3 was 35 (83-184) mg/dL and C4 was 6 (17-59) $\mathrm{mg} / \mathrm{dL}$. ADAMTS13 activity was adequate at $63 \%$. Stool studies were all negative (including Escherichia coli 0157:H7 and Clostridium difficile). She underwent colectomy and was confirmed to have scattered fibrin microthrombi on her bowel specimen. Her renal failure worsened and continuous venovenous hemodialysis was initiated. A rapid neurologic decline occurred after surgery. Severe renal failure, profound neurologic changes, severe hemolysis, thrombotic microangiopathy, and low complement levels were all suggestive of atypical hemolytic uremic syndrome (aHUS). By hospital day 5, there were occasional schistocytes on the peripheral smear. Since aHUS is characterized by chronic systemic uncontrolled complement activation, ${ }^{2}$ the anti-C5 monoclonal antibody eculizumab (900 mg IV) was administered on hospital day 6. It was continued weekly for four doses, followed by maintenance therapy that was gradually reduced. ${ }^{16}$

Because eculizumab increases the risk of Neisseria meningitidis, the meningococcal vaccine was given prior to the first dose, and 14 days of ciprofloxacin was prescribed for meningococcal prophylaxis. ${ }^{16}$

Her renal recovery was remarkable with eculizumab and she has remained off dialysis with long-term eculizumab treatment. Our patient's rapid and profound neurologic deterioration resulted from significant thrombotic microangiopathy. MRI showed a right parietal lobe infarction (images shown in original paper). Despite thrombotic microangiopathic brain injury, she had a swift and complete neurologic recovery with eculizumab.

The initial dosing schema used for our patient is similar to that used in the clinical trials of eculizumab in aHUS, but maintenance therapy has gradually been reduced over time to a dose lower than what is used in the trials. Maintenance therapy, starting on week 5, was planned to be $1200 \mathrm{mg}$ every 2 weeks, as in the trials; however, this dose was only continued for doses five and six. ${ }^{17-19}$ Due to nausea at the $1200 \mathrm{mg}$ dose, the seventh dose was delayed by 1 day because the patient refused it, but then agreed to a reduced dose of $600 \mathrm{mg}$ weekly. Her creatinine continued to improve despite the dose modification. She then remained on $600 \mathrm{mg}$ weekly for nine doses with stable renal function. Her dose was subsequently reduced to $600 \mathrm{mg}$ every 2 weeks and her improved renal function has been maintained despite the dose reduction. Additionally, her creatinine has continued to decrease on maintenance therapy with recent levels as low as $1.64 \mathrm{mg} / \mathrm{dL}$ (normal $0.5-1.3 \mathrm{mg} / \mathrm{dL}$ ), despite maintenance dosing reduced to $600 \mathrm{mg}$ every 2 weeks. Figures $1-6$ show treatment responses over time with respect to creatinine, $\mathrm{C} 3$, $\mathrm{C} 4, \mathrm{LDH}$, haptoglobin, and platelets.

\section{Discussion}

\section{Maintenance eculizumab}

Several authors have described how long-term eculizumab therapy helps avoid aHUS relapse. ${ }^{1-6}$ Mache et al describe how aHUS relapsed after discontinuation of eculizumab and resulted anuric end stage renal disease. ${ }^{1}$ They describe another patient who experienced improvement in renal function, as well as normalization of platelets and haptoglobin, within 5 days of receiving eculizumab $600 \mathrm{mg}$. Unfortunately, atypical hemolytic uremic syndrome relapsed 2 weeks after recovery of complement-mediated hemolytic

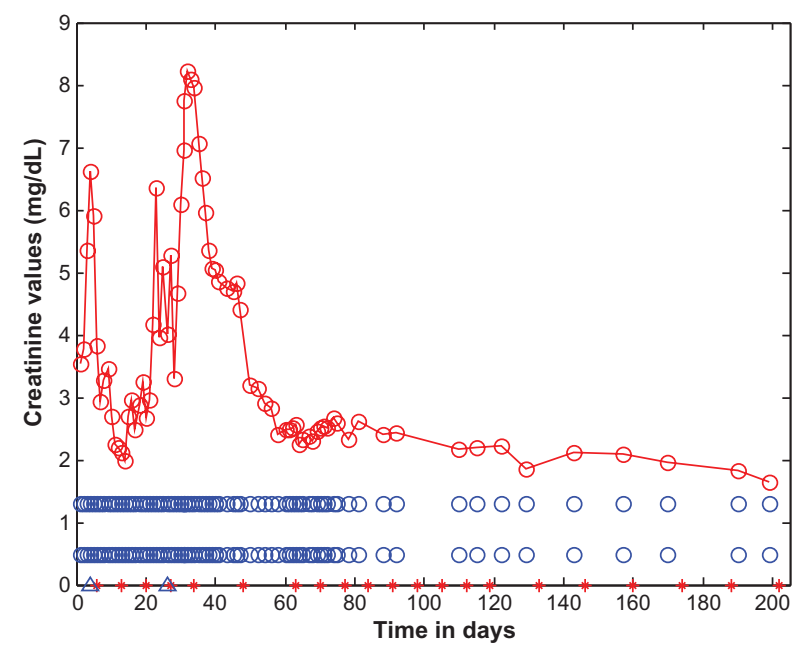

Figure I Serum creatinine levels as a function of time (days).

Notes: Creatinine levels are marked with open red circles and the data points are connected with straight lines. For the purposes of reference, the normal creatinine range $(0.5-1.3 \mathrm{mg} / \mathrm{dL})$ is shown as open blue circles. The days corresponding to eculizumab administration are indicated by red asterisks along the horizontal time axis, appearing on days: $6,13,20,27,34,48,63,70,77,84,91,98,105,112,119,133$, $146,160,174,188$, and 202. The start and end dates of dialyses administration are marked on the time axis of the figure by two blue open triangles, on days 4 and 26 . 


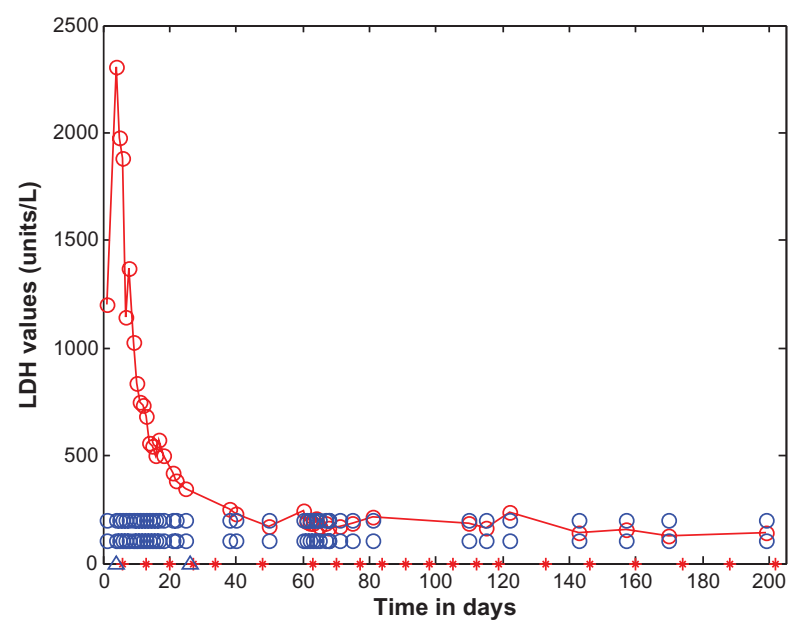

Figure 2 Lactate dehydrogenase as a function of time (days).

Notes: Lactate dehydrogenase levels are marked with open red circles and the data points are connected with straight lines. For the purposes of reference, the normal range (108-202 U/L) is shown as open blue circles. Lactate dehydrogenase is shown as a function of time (days). Lactate dehydrogenase gradually dropped after initiating treatment with eculizumab. Eculizumab administration is denoted by vertical tick marks (days 6, 13, 20, 27, 34, 48, 63, 70, 77, 84, 91, 98, 105, II2, II9, I33, I46, I60, 174,188 , and 202). The start and end dates of dialyses administration are marked on the time axis of the figure by two blue open triangles, on days 4 and 26 . Four days after the first dose, lactate dehydrogenase dropped to $837 \mathrm{U} / \mathrm{L}$. After the second dose of eculizumab on day 13, lactate dehydrogenase dropped to $556 \mathrm{U} / \mathrm{L}$. A week after the third dose, lactate dehydrogenase was down to $349 \mathrm{U} / \mathrm{L}$. Three days after the sixth dose, lactate dehydrogenase was normal. It did rise slightly before the seventh dose was due.

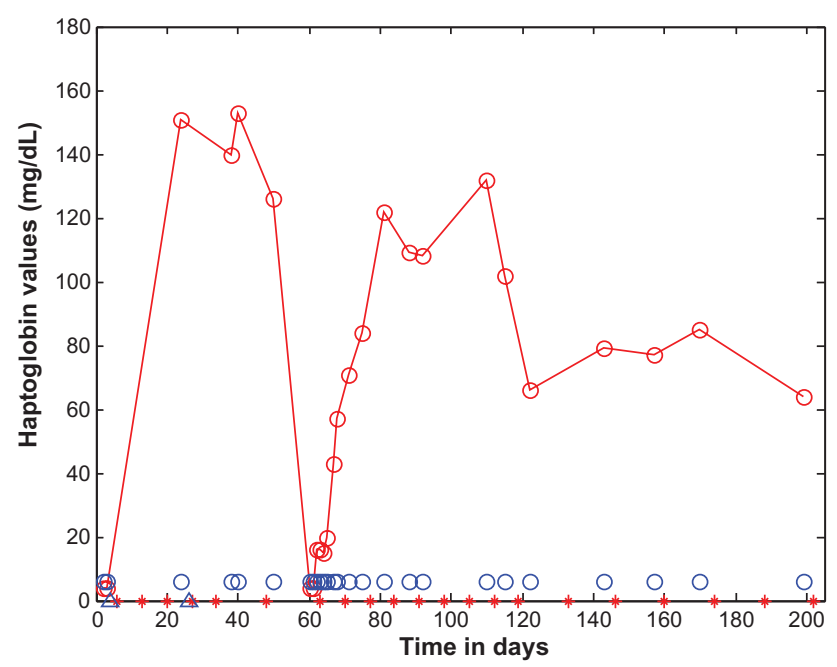

Figure 3 Haptoglobin as a function of time (days).

Notes: Haptoglobin levels are marked with open red circles and the data points are connected with straight lines. For the purposes of reference, the normal range $(62-197 \mathrm{mg} / \mathrm{dL})$ is shown as open blue circles. Eculizumab administration is denoted by vertical tick marks (days $6,13,20,27,34,48,63,70,77,84,91,98,105,112$, $119,133,146,160,174,188$, and 202). Initial haptoglobin was $<6 \mathrm{mg} / \mathrm{dL}$ (normal range $62-197 \mathrm{mg} / \mathrm{dL}$ ). Five days after the third dose, haptoglobin was normal and remained normal until the time the seventh dose was due. Haptoglobin then dropped precipitously, indicating an ongoing need for the drug. Haptoglobin did increase again after the seventh dose on day 63 , and later normalized to $71 \mathrm{mg} / \mathrm{dL}$ 3 days after receiving the eighth dose on day 69 . The start and end dates of dialyses administration are marked on the time axis of the figure by two blue open triangles, on the days 4 and 26 .

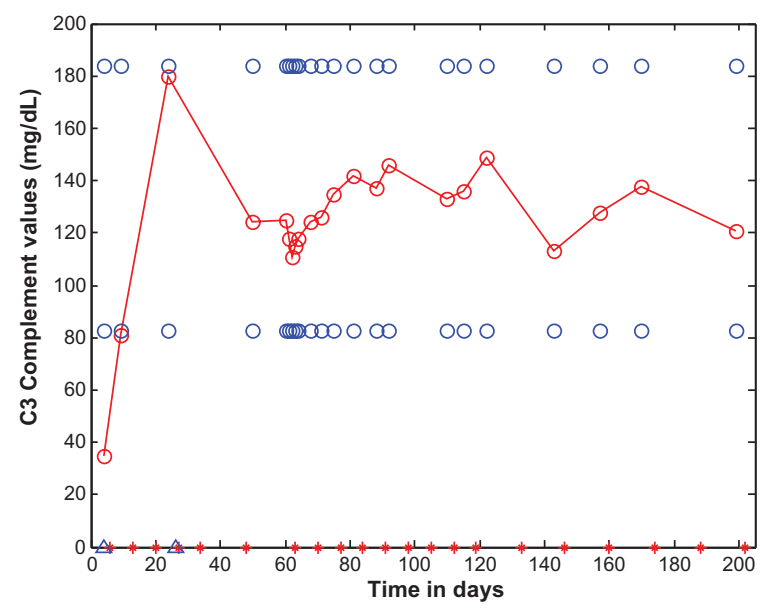

Figure $4 \mathrm{C} 3$ as a function of time (days).

Notes: C3 levels are marked with open red circles and the data points are connected with straight lines. For the purposes of reference, the normal range $(83-184 \mathrm{mg} / \mathrm{dL})$ is shown as open blue circles. C3 is shown as a function of time (days). Eculizumab administration is denoted by vertical tick marks (days 6, 13, 20, $27,34,48,63,70,77,84,91,98,105,112,119,133,146,160,174,188$, and 202). The start and end dates of dialyses administration are marked on the time axis of the figure by two blue open triangles, on the days 4 and 26. After the second dose of eculizumab on day 13, C3 rose to 81. Thirteen days after the sixth dose, C3 was normal and remained normal.

activity, suggesting the need for sustained complement inhibition to prevent relapse.

Châtelet et al described a transplant patient who was on a maintenance eculizumab $1200 \mathrm{mg}$ every 14 days. ${ }^{2}$ They reported that a 6-day delay in administering the ninth dose resulted in recurrent hemolysis and deterioration of transplant function. The authors consequently resumed eculizumab as permanent therapy for their patient, with recovery of renal function. Similarly, in our patient, when the seventh dose was due, laboratory investigations suggested relapsing hemolysis, with haptoglobin $<6 \mathrm{mg} / \mathrm{dL}$, indicating her continued need for the drug.

Zuber et al mention similar cases which emphasize the importance of long-term eculizumab therapy. ${ }^{3}$ They describe two post-transplant aHUS cases in which a single dose of eculizumab resulted in long-term remission in both patients. However, in both cases relapse ultimately occurred. Relapse occurred after 11.5 months for one patient and eculizumab was resumed, resulting in a remission. The other patient relapsed after 21 months, but when eculizumab was resumed, graft loss could be not prevented. Zuber et al suggest that a single dose of eculizumab is less effective than prolonged therapy and argue that the interval between doses be no more than 14 days. ${ }^{3}$

\section{Genetic testing in aHUS}

Approximately $60 \%$ of aHUS cases (familial and sporadic) have been associated with genetic mutations in complement 


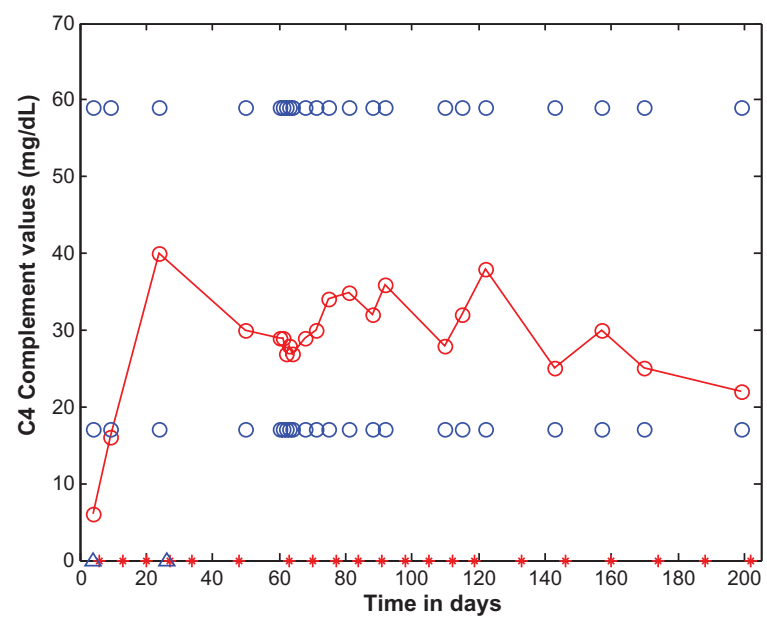

Figure $5 \mathrm{C} 4$ as a function of time (days).

Notes: $\mathrm{C} 4$ levels are marked with open red circles and the data points are connected with straight lines. For the purposes of reference, the normal range ( 17 to $59 \mathrm{mg} / \mathrm{dL}$ ) is shown as open blue circles. C4 is shown as a function of time (days). Eculizumab administration is denoted by vertical tick marks (days $6,13,20,27,34,48,63,70$, $77,84,91,98,105,112,119,133,146,160,174,188$, and 202). The start and end dates of dialyses administration are marked on the time axis of the figure by two blue open triangles, on days 4 and 26. The day after the second dose of eculizumab on day 13, C4 rose to 16. Three days after the sixth dose, C4 was normal and remained normal.

regulatory proteins of the alternative complement pathway or auto-antibodies to the complement regulatory factor $\mathrm{CFH} .{ }^{19,20}$ Consequently, we ordered the following genetic and antibody tests for our patient: $C F H, C F B, C F I, C F H R 3-C F H R 1$ (deletion testing), $M C P, T H B D, C 3$, and CFH auto-antibodies (serum test). The Complement Hemolytic assay (serum test) was also ordered. All these results were normal for our patient.

As gene sequencing is very time consuming, it can take several months for all of the genetic tests to return. In the case of our patient, all of the results were available 6 months after they were ordered. Furthermore, very few clinical laboratories do genetic testing for aHUS, but such labs may be found on the National Center for Biotechnology Information (NCBI) website: http://www.genetests.org/. ${ }^{21}$ Our patient's genetic tests were performed at University of Iowa, which, to our knowledge, may be the only lab in the US that does these tests. 22

Atypical HUS has been linked to the chromosome 1q32 locus containing genes for complement factor $\mathrm{H}(\mathrm{CFH})$, the plasma regulator of the alternative complement pathway, as well as for other complement regulators. Over $80 \mathrm{CFH}$ mutations have been identified in aHUS patients. In familial aHUS the frequency of $\mathrm{CFH}$ mutations is $40 \%-50 \%$. In the sporadic form, the frequency of CFH mutations is $20 \%{ }^{19,20}$ The various genetic abnormalities and antibody abnormalities currently identified in aHUS are summarized below. More information

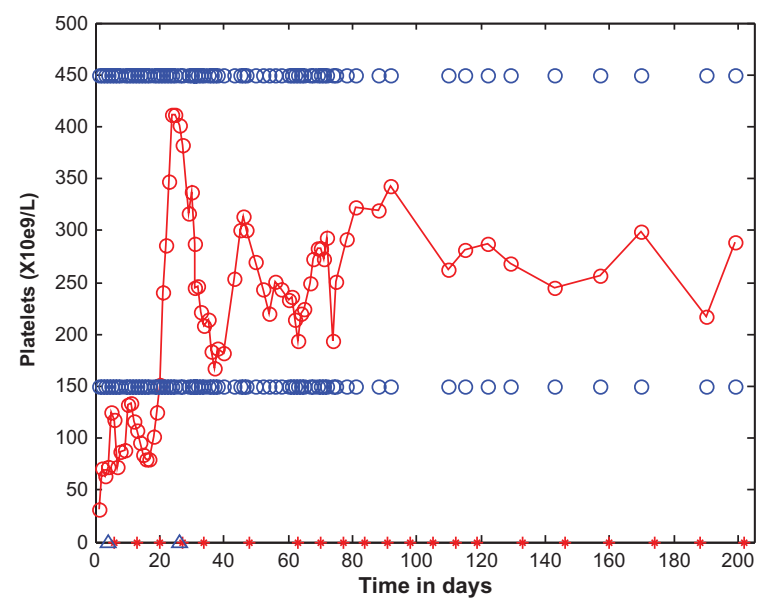

Figure 6 Platelets as a function of time (days).

Notes: Platelet levels are marked with open red circles and the data points are connected with straight lines. For the purposes of reference, the normal range $\left(150-450 \times 10^{9} / \mathrm{L}\right)$ is shown as open blue circles. The days corresponding to eculizumab administration are indicated by red asterisks along the horizontal time axis, appearing on days: $6,13,20$, $27,34,48,63,70,77,84,91,98,105,112,119,133,146,160,174,188$, and 202. On the day of transfer, hospital day I, the patient underwent total abdominal colectomy. During surgery, she received $2 U$ of pooled platelets. Initial platelets before surgery were $28 \times 10^{\circ} / \mathrm{L}$. After surgery, her platelets rose, and continued to rise during the first 5 days of the admission, without additional transfusions. The rising platelets after colectomy suggested previous platelet consumption by a thrombotic microangiopathic process in the colon. After initiating eculizumab, her platelets continued to rise.

on genetic mutations in aHUS can be obtained from two comprehensive papers by Noris and Remuzzi. 19,20 $^{2}$

- $C F H$ regulates the alternative complement pathway (AP). In aHUS, most $C F H$ mutations are heterozygous. ${ }^{19,20}$ Mutant CFH heterozygotes usually carry normal levels of $\mathrm{CFH}$ but $50 \%$ of the molecules are dysfunctional. Only $15 \%-20 \%$ of $\mathrm{CFH}$ mutations in aHUS are homozygous, resulting in quantitative $\mathrm{CFH}$ deficiency and very low $\mathrm{C} 3$ levels. ${ }^{19,20}$

- $3 \%-5 \%$ of aHUS cases possess a heterozygous hybrid gene. The hybrid gene results from aberrant crossover between $C F H$ and $C F H R 1$, a gene located in tandem to $C F H$. CFHR1 encodes complement factor $\mathrm{H}$ related protein (CFHR1). The gene product has decreased endothelial complement regulatory activity. ${ }^{19,20}$

- Deletion testing for CFHR1 and CFHR3 was ordered because these gene deletions are considered a risk factor for aHUS. ${ }^{23}$

- Approximately $6 \%-10 \%$ of aHUS patients acquire anti$\mathrm{CFH}$ antibodies, which emulate $\mathrm{CFH}$ mutations by binding to the $\mathrm{CFH} \mathrm{C}$-terminus and decreasing $\mathrm{CFH}$ binding to $\mathrm{C} 3 \mathrm{~b}$ and surface cofactor activity. ${ }^{19,20}$ Furthermore, some aHUS patients acquire autoantibodies to Factor $\mathrm{H}$ in the setting of deletions of CFHR 1 and CFHR3. ${ }^{24}$

- CFI is a plasma serine protease that regulates all three complement pathways by cleaving $\mathrm{C} 3 \mathrm{~b}$ and $\mathrm{C} 4 \mathrm{~b}$. 
$4 \%-10 \%$ of aHUS cases involve a heterozygously mutated CFI. ${ }^{19,20}$ As a proteolytic enzyme, CFI prevents hemolytic and immune-adherence activities of activated, cell-bound $\mathrm{C} 3 .{ }^{22}$

- $M C P$ encodes the membrane cofactor protein (MCP), which is a cofactor for CFI to cleave $\mathrm{C} 3 \mathrm{~b}$ and $\mathrm{C} 4 \mathrm{~b}$ on cell surfaces. Around $10 \%$ of aHUS cases involve $M C P$ mutations, the majority of which are heterozygous. ${ }^{19,20}$

- Some patients have gain of function mutations that affect genes encoding $\mathrm{CFB}$ and $\mathrm{C} 3$, which are the AP $\mathrm{C} 3$ convertase components. $C F B$ mutations, accounting for only $1 \%-2 \%$ of aHUS cases, result in chronic AP activation with low $\mathrm{C} 3$ level and normal $\mathrm{C} 4$ levels. ${ }^{19,20}$ Approximately $4 \%-10 \%$ of aHUS cases have heterozygously mutated $C 3$, associated with reduced $\mathrm{C} 3 \mathrm{~b}$ binding to $\mathrm{CFH}$ and $\mathrm{MCP}$, significantly hindering degradation of mutant $\mathrm{C} 3 \mathrm{~b}$ and resulting in low $\mathrm{C} 3$ levels. ${ }^{19,20}$

- Around $5 \%$ of aHUS patients have heterozygously mutated $T H B D$, resulting in inefficient inactivation of C $3 \mathrm{~b}$. THBD encodes thrombomodulin that is involved in complement activation on cell surfaces..$^{19,20}$ Thrombomodulin is actually a negative regulator of the complement system, which suppresses leukocyte trafficking, inflammation, and complement activation. ${ }^{22,25}$

- Of note, $3 \%-5 \%$ of aHUS patients possess combined mutations, often in $C F H$ along with either $M C P$ or $C F I$. Few also possess anti-CFH antibodies..$^{19,20}$

Although genetic testing did not change the management of our patient, genetic testing in aHUS can provide the clinician with information about disease process and long-term renal transplant outcome. ${ }^{19,20}$ For example, mutations of $\mathrm{CFH}$, $C F I, C F B$, and $C 3$ have been associated with post-transplant recurrence. This is due to the fact that the mutations give rise to abnormalities in circulating proteins that mostly come from the liver, and thus can exist even after kidney transplant. ${ }^{19,20}$

\section{Conclusion}

Six months after initial diagnosis, our patient continues to have improved renal function on maintenance doses of eculizumab as low as $600 \mathrm{mg}$ every 2 weeks. This dose is much lower than what is used in the current clinical trials, suggesting that complement inhibition needs vary among patients. Clinical trials are underway to determine the optimal dosing schema.

Although genetic abnormalities in complement regulatory factors or anti-CFH antibodies have been identified in approximately $60 \%$ of cases, ${ }^{19,20}$ genetic testing did not change the management of this patient.

\section{Acknowledgment}

The authors would like to acknowledge Camille Bedrosian, MD, for her important recommendations on the treatment of aHUS.

\section{Disclosure}

The authors report no conflicts of interest in this work.

\section{References}

1. Mache CJ, Acham-Roschitz B, Frémeaux-Bacchi V, et al. Complement inhibitor eculizumab in atypical hemolytic uremic syndrome. Clin J Am Soc Nephrol. 2009;4(8):1312-1316.

2. Châtelet V, Lobbedez T, Frémeaux-Bacchi V, Ficheux M, Ryckelynck JP, Hurault de LB. Eculizumab: safety and efficacy after 17 months of treatment in a renal transplant patient with recurrent atypical hemolytic-uremic syndrome: case report. Transplant Proc. 2010;42(10): 4353-4355.

3. Zuber J, Le Quintrec M, Sberro-Soussan R, Loirat C, Frémeaux-Bacchi V, Legendre C. New insights into postrenal transplant hemolytic uremic syndrome. Nat Rev Nephrol. 2011;7(1):23-35.

4. Nurnberger J, Phillip T, Witzke O, et al. Eculizumab for atypical hemolytic-uremic syndrome. N Engl J Med. 2009;360(5):542-544.

5. Châtelet V, Frémeaux-Bacchi V, Lobbedez T, Ficheux M, Hurault de LB Safety and long-term efficacy of eculizumab in a renal transplant patient with recurrent atypical hemolytic-uremic syndrome. Am J Transplant. 2009;9(11):2644-2645.

6. Gruppo RA, Rother RP. Eculizumab for congenital atypical hemolyticuremic syndrome. N Engl J Med. 2009;360(5):544-546.

7. Davin JC, Gracchi V, Bouts A, Groothoff J, Strain L, Goodship T. Maintenance of kidney function following treatment with eculizumab and discontinuation of plasma exchange after a third kidney transplant for atypical hemolytic uremic syndrome associated with a $\mathrm{CFH}$ mutation. Am J Kidney Dis. 2010;55(44):708-711.

8. Zimmerhackl LB, Hofer J, Cortina G, et al. Prophylactic eculizumab after renal transplantation in atypical hemolytic-uremic syndrome. N Engl J Med. 2010;362(18):1746-1748.

9. Ardissino G, Testa S, Paglialonga F, et al. Remission of plasma-resistant atypical hemolytic uremic syndrome relapse on kidney graft with eculizumab. Presented at the 2nd International Conference on HUSMPGN-PNH; June 13-15, 2010; Innsbruck, Austria. p. 17.

10. Haffner H, Hofer J, Zimmerhackl LB, Pohl M. Effective eculizumab therapy of familiar atypical HUS in a 4 year old patient. Presented at the 2nd International Conference on HUS-MPGN-PNH; 2010 June 13-15; Innsbruck, Austria. p. 21.

11. Lapayraque AL, Phan V, Clermont MJ, et al. Effectiveness of eculizumab in a plasma infusion dependent patient with atypical haemolytic and uremic syndrome (a HUS) associated with heterozygous combined de Novo mutation in factor $\mathrm{H}$ gene. Presented at the 2nd International Conference on HUS-MPGN-PNH; June 13-15, 2010; Innsbruck, Austria. p. 24.

12. Tschumi S, Bucher BS, Sparta G, et al. Eculizumab in atypical hemolytic uremic syndrome: long-term clinical course and histological findings. Presented at the 2nd International Conference on HUS-MPGN-PNH; June 13-15, 2010; Innsbruck, Austria. p. 44.

13. Larrea CF, Cofan F, Oppenheimer F, Campistol JM, Escolar G, Lozano M. Efficacy of eculizumab in the treatment of recurrent atypical hemolytic-uremic syndrome after renal transplantation. Transplantation . 2010;89(7):903-904.

14. Legault DJ, Boelkins MR. Successful treatment of aHUS recurrence and arrest of plasma exchange resistant TMA post-renal transplantation with the terminal complement inhibitor eculizumab. Presented at the 51st ASH Annual meeting; December 6, 2009; Grand Rapids, MI. p. 2421 . 
15. Dmytrijuk A, Robie-Suh K, Cohen MH, Rieves D, Weiss K, Pazdur R. FDA report: eculizumab (Soliris) for the treatment of patients with paroxysmal nocturnal hemoglobinuria. Oncologist. 2008;13(9):993-1000.

16. Ohanian M, Cable C, Halka K. Eculizumab safely reverses neurologic impairment and eliminates need for dialysis in severe atypical hemolytic uremic syndrome. Clinical Pharmacology: Advances and Applications. doi: 10.2147/CPAA.S17904.

17. Alexion Pharmaceuticals. Open Label Controlled Trial of Eculizumab in Adult Patients With Plasma Therapy-sensitive Atypical Hemolytic Uremic Syndrome (aHUS). In: ClinicalTrials.gov [Internet]. Bethesda, MD: National Library of Medicine(US). 2009. Available from: http://www.clinicaltrials.gov/ct2/show/NCT00838513?term=NCT00838513\&rank=1. Accessed on October 5, 2011. NLM identifier: NCT00838513.

18. Muus P, Legendre C, Douglas K, Hourmant M, Delmas Y, Herthelius B, et al. Safety and Efficacy of Eculizumab in aHUS Patients on Chronic Plasma Therapy: Interim Analysis from a Phase II Trial [abstract]. Presented at American Society of Nephrology Meeting, Renal Week 2010, Denver, CO. Available at: http://www.abstracts2view.com/asn10/ view.php?nu=ASN10L1_1338a. Accessed on October 5, 2011.
19. Noris M, Remuzzi G. Genetics and genetic testing in hemolytic uremic syndrome/thrombotic thrombocytopenic purpura. Semin Nephrol. 2010; 30(4):395-408.

20. Noris M, Remuzzi G. Atypical hemolytic-uremic syndrome. $N$ Engl J Med. 2009;361(17):1676-1687.

21. GeneTests [homepage]. Available at: http://www.ncbi.nlm.nih.gov/ sites/GeneTests. Accessed on October 5, 2011.

22. Molecular Otolaryngology and Renal Research Laboratories [homepage]. Available at: http://www.healthcare.uiowa.edu/labs/morl/THBD.htm. Accessed on October 5, 2011.

23. Zipfel PF, Edey M, Heinen S, et al. Deletion of complement factor H-related genes CFHR1 and CFHR3 is associated with atypical hemolytic uremic syndrome. PLoS Genet. 2007;3(3):e41.

24. Skerka C, Józsi M, Zipfel PF, Dragon-Durey MA, Frémeaux-Bacchi V. Autoantibodies in haemolytic uraemic syndrome (HUS). Thromb Haemost. 2009;101(2):227-232.

25. Delvaeye M, Noris M, De Vriese A, et al. Thrombomodulin mutations in atypical hemolytic-uremic syndrome. $N$ Engl J Med. 2009;361(4): 345-357.

\section{Publish your work in this journal}

Clinical Pharmacology: Advances and Applications is an international, peer-reviewed, open access journal publishing original research, reports, reviews and commentaries on all areas of drug experience in humans. The manuscript management system is completely online and includes a very quick and fair peer-review system, which is all easy to use.
Visit http://www.dovepress.com/testimonials.php to read real quotes from published authors. 